\title{
Estimativas de parâmetros genéticos para produção de leite e persistência da lactação em vacas Gir, aplicando modelos de regressão aleatória
}

\section{Luis Gabriel González Herrera ${ }^{1}$, Lenira El Faro², Lucia Galvão de Albuquerque ${ }^{3}$, Humberto Tonhati $^{3}$, Carlos Henrique Cavallari Machado 5}

${ }^{1}$ FCAVIUNESP, Rod. Prof. Paulo D. Castelani km 8, CEP: 14870-000, Jaboticabal - SP

2 APTA/SAA/SP, Ribeirão Preto - SP.

${ }^{3}$ FCAVIUNESP, Jaboticabal - SP. Pesquisador do CNPq

${ }^{4}$ ABCZ - Superintendente técnico-adjunto de Melhoramento Genético.

RESUMO - Com o objetivo de estimar parâmetros genéticos para a produção de leite no dia do controle (PLDC), foram usadas as 2.440 primeiras lactações de vacas da raça Gir leiteira, com partos registrados entre 1990 e 2005. As PLDC foram consideradas em dez classes mensais e analisadas por meio de modelo de regressão aleatória (MRA) utilizando-se como efeitos aleatórios o genéticoaditivo, o de ambiente permanente e o residual e, como efeitos fixos, o grupo de contemporâneos (GC), a co-variável idade da vaca ao parto (efeito linear e quadrático) e a curva média de lactação da população. Os efeitos genético-aditivos e de ambiente permanente foram modelados utilizando-se as funções de Wilmink (WIL) e Ali e Schaeffer (AS). As variâncias residuais foram modeladas utilizando-se 1, 4, 6 ou 10 classes. Os grupos de contemporâneos foram definidos como rebanho-ano-estação do controle contendo no mínimo três animais. Os testes indicaram que o modelo com quatro classes de variâncias usando a função paramétrica AS foi o que melhor se ajustou aos dados. As estimativas de herdabilidade variaram de 0,21 a 0,33 para a função AS e de 0,17 a 0,30 para WIL e foram maiores na primeira metade da lactação. As correlações genéticas entre as PLDC foram positivas e elevadas entre os controles adjacentes e diminuiram quando a distância entre os controles aumentou. Para o melhor modelo, foram estimados os valores genéticos para a produção de leite acumulada até os 305 dias e, para períodos parciais da lactação, foram obtidas como médias dos valores genéticos preditos naquele período. Os valores genéticos foram comparados, por meio da correlação de posto, ao valor genético predito para a produção acumulada até os 305 dias, pelo método tradicional. As correlações entre os valores genéticos indicaram que podem ocorrer divergências na classificação dos animais pelos critérios estudados.

Palavras-chave: funções de co-variância, função paramétrica, parâmetros genéticos, regressão aleatória

\section{Estimates of genetic parameters for milk yield and persistency of lactation of Gyr cows, applying random regression models}

\begin{abstract}
Data consisting of 2,440 first lactations of Gyr cows calving between 1990 and 2005 were used to estimate genetic parameters of monthly test-day milk yield (TDMY). Records were analyzed by random regression models (MRA) that included the additive genetic and permanent environmental random effects and the contemporary group, age of cow at calving (linear and quadratic components) and the average trend of the population as fixed effects. Random trajectories were fitted by Wilmink's (WIL) and Ali \& Schaeffer's (AS) parametric functions. Residual variances were fitted by step functions with 1, 4, 6 or 10 classes. The contemporary group was defined by herd-year-season of test-day and included at least three animals. Models were compared by Akaike's and Schwarz's Bayesian (BIC) information criterion. The AS function used for modeling the additive genetic and permanent environmental effects with heterogeneous residual variances adjusted with a step function with four classes was the best fitted model. Heritability estimates ranged from 0.21 to 0.33 for the AS function and from 0.17 to 0.30 for WIL function and were larger in the first half of the lactation period. Genetic correlations between TDMY were high and positive for adjacent test-days and decreased as days between records increased. Predicted breeding values for total 305-day milk yield (MRA305) and specific periods of lactation (obtained by the mean of all breeding values in the periods) using the AS function were compared with that predicted by a standard model using accumulated 305-day milk yield (PTA305) by rank correlation. The magnitude of correlations suggested differences may be observed in ranking animals by using the different criteria which were compared in this study.
\end{abstract}

Key Words: covariance functions, genetic parameters, parametric function, random regression

Este artigo foi recebido em 23/8/2007 e aprovado em 19/3/2008.

Correspondências devem ser enviadas para pascu002000@yahoo.es. 


\section{Introdução}

Segundo Schaeffer (2004), a maior aplicação dos modelos de regressão aleatória tem sido na avaliação genética de bovinos leiteiros e na análise das produções de leite no dia do controle (PLDC). Esses modelos possibilitam a predição de valores genéticos para a produção de leite acumulada na lactação para qualquer dia em lactação e para funções da curva de lactação, diferentemente dos modelos multicaracterísticos, que possibilitam apenas predições pontuais.

O uso dos modelos de regressão aleatória no melhoramento animal foi proposto, inicialmente, por Henderson Jr. (1982) e, posteriormente, por Schaeffer \& Dekkers (1994), para avaliação genética de bovinos leiteiros. Com esses modelos, é possível ajustar uma curva de lactação aleatória para cada indivíduo, expressa como desvio de uma curva média da população ou de grupos de indivíduos (Schaeffer, 1996). As regressões são ajustadas por meio de funções lineares e modelam trajetórias para a média populacional (regressões fixas) e para cada animal (regressão aleatória).

As matrizes de delineamentos dos efeitos aleatórios para os modelos de regressão aleatória, por conterem covariáveis nos seus elementos, são, portanto, diferentes dos zeros e uns dos modelos convencionais; são mais densas, o que exige maior capacidade computacional (Van Der Werf \& Schaeffer, 1998).

Em vários trabalhos com bovinos, foram aplicados modelos de regressão aleatória para modelar a produção de leite no dia do controle. Os resultados indicaram que as estimativas de herdabilidade por estes modelos foram maiores que as obtidas pelos modelos multicaraterísticos e geralmente foram mais elevadas no início e ao final da lactação (Olori et al., 1999; Brotherstone et al., 2000; El Faro \& Albuquerque, 2003).

$\mathrm{Na}$ literatura existem poucos trabalhos aplicando os modelos de regressão aleatória para as produções no dia do controle de vacas da raça Gir. Costa et al. (2005) estimaram componentes de variância e parâmetros genéticos para as PLDC usando modelos de regressão aleatória sob polinômios ortogonais de Legendre e duas funções paramétricas já conhecidas na literatura para o ajuste de curvas de lactação: a função logarítmica (Ali \& Schaeffer, 1987) e a exponencial (Wilmink, 1987). Esses autores encontraram estimativas de herdabilidade altas para as produções de leite, principalmente no início da lactação. As estimativas de herdabilidade para ambas as funções paramétricas variaram de 0,49 a 0,82 nos primeiros 60 dias de lactação, chegando a 0,20 no final da lactação. Para os modelos com polinômios ortogonais, as herdabilidades também foram altas e variaram de 0,71 no início a 0,30 no final da lactação.

Entre as aplicações dos modelos de regressão aleatória, está a possibilidade de predição de valores genéticos para determinadas fases da lactação, para a produção acumulada aos 305 dias e para características de importância econômica, por exemplo, a persistência da lactação. A importância da avaliação genética desta característica está na necessidade de encontrar vacas com curvas de lactação com menor taxa de declínio após o pico, pois espera-se que vacas mais persistentes sejam mais viáveis economicamente (Ludwich \& Petersen, 1943; Dekkers et al., 1998). A persistência é uma característica de grande relevância para a raça Gir, pois animais dessa raça tendem a apresentar curvas com baixa persistência e lactações menos duradouras, o que torna interessante a aplicação de modelos que permitam avaliá-la (Costa et al., 2005). Em diversos estudos, ficou evidente a existência de diferenças genéticas para persistência da lactação entre animais (Sölkner \& Fuchs, 1987; Swalve, 1995; Jamrozik et al., 1997; Tekerli et al., 2000).

Objetivou-se neste estudo estimar parâmetros genéticos para as produções mensais de leite no dia do controle de vacas da raça Gir, aplicando modelos de regressão aleatória, e verificar a possibilidade de usar a persistência de lactação como característica a ser incluída nas avaliações genéticas de animais dessa raça.

\section{Material e Métodos}

Foram analisados 14.035 controles leiteiros mensais de produção de leite de 2.440 primeiras lactações de vacas da raça Gir leiteiro, pertencentes a rebanhos controlados pela Associação Brasileira de Criadores de Gado Zebu (ABCZ). Os dados analisados foram provenientes de animais de 24 a 61 meses de idade, com partos registrados entre os anos de 1990 e 2005, de 119 rebanhos de diversas regiões do Brasil. As lactações foram registradas a partir do quinto dia de lactação e foram truncadas aos 305 dias. As PLDC foram agrupadas em dez classes mensais de lactação (Tabela 1) e analisadas por meio de um modelo de regressão aleatória (MRA), unicaracterístico, sob um modelo animal que incluiu o grupo de contemporâneos como efeito fixo e as co-variáveis idade da vaca ao parto como efeitos linear e quadrático, além da curva média de lactação da população. Os efeitos aleatórios, genético aditivo e de ambiente permanente de animal foram modelados por meio das funções paramétricas de Wilmink (1987) e Ali \& Schaeffer (1987), assim como a trajetória fixa. $\mathrm{O}$ grupo de contemporâneos foi definido pelas variáveis rebanho, ano e estação do controle, contendo 
773 níveis, impondo-se a condição de que cada grupo de contemporâneos deveria ter no mínimo três observações. Em todas as análises, utilizou-se um arquivo de pedigree contendo identificação de animal, pai e mãe e, depois de buscar o parentesco entre os animais por três gerações, totalizaram-se 7.582 animais na matriz de parentesco.

A estrutura de variâncias residuais foi considerada homogênea (contendo uma variância) e, quando heterogênea, por meio de classes, contendo quatro, seis ou dez classes de variâncias. Quando se empregaram estruturas com quatro ou seis classes heterogêneas, as variâncias foram agrupadas de acordo com o comportamento da variação no decorrer da lactação, com base nos modelos contendo dez classes de variâncias. Para os modelos que continham quatro classes, os meses de lactação foram agrupados da seguinte forma: 1, 2-5, 6-9 e 10 meses. Para o modelo contendo seis classes, os meses foram agrupados como 1, 2-4, 5, 6, 7-9 e 10.

O modelo de regressão aleatória geral utilizado pode ser representado por:

$\mathrm{y}_{\mathrm{ij}}=\mathrm{F}+\sum_{\mathrm{m}=0}^{\mathrm{k}_{\mathrm{b}}-1} \beta_{\mathrm{m}} \phi_{\mathrm{m}}\left(\mathrm{t}_{\mathrm{i}}\right)+\sum_{\mathrm{m}=0}^{\mathrm{k}_{\mathrm{a}}-1} \alpha_{\mathrm{jm}} \phi_{\mathrm{m}}\left(\mathrm{t}_{\mathrm{ij}}\right)+\sum_{\mathrm{m}=0}^{\mathrm{k}_{\mathrm{ap}}-1} \gamma_{\mathrm{jm}} \phi_{\mathrm{m}}\left(\mathrm{t}_{\mathrm{ij}}\right)+\varepsilon_{\mathrm{ij}}$ em que: $y_{i j}=$ produção de leite da i-ésima classe de dia em lactação, pertencente ao j-ésimo animal; $F$ = conjunto de efeitos fixos, exceto as covariáveis dia de lactação sobre a produção de leite; $\mathrm{b}_{\mathrm{m}}=$ conjunto de $\mathrm{m}$ regressores fixos para a trajetória média da população; $\phi_{\mathrm{m}}\left(\mathrm{t}_{\mathrm{i}}\right)=$ função de regressão de ordem $\mathrm{k}_{\mathrm{b}}$, que descreve a trajetória média para cada ordem de parto, de acordo com o dia de lactação $\left(\mathrm{t}_{\mathrm{i}}\right) ; \phi_{\mathrm{m}}\left(\mathrm{t}_{\mathrm{ij}}\right)=$ funções de regressão, que descrevem as trajetórias de cada indivíduo $\mathrm{j}$, de acordo com o dia de lactação $\left(\mathrm{t}_{\mathrm{j}}\right)$ para os efeitos aleatórios genético aditivos e de ambiente permanente; $\alpha_{j m}, \gamma_{j m}=$ m regressores aleatórios genético-aditivos e de ambiente permanente para cada animal $\mathrm{j} ; \mathrm{k}_{\mathrm{b}}, \mathrm{k}_{\mathrm{a}}$ e $\mathrm{k}_{\mathrm{ap}}=$ ordens das funções lineares utilizadas para a trajetória média e para os efeitos genético aditivos e de ambiente permanente, respectivamente; e $\varepsilon_{i j}=$ erro aleatório NID associado a cada controle i do animal j.

A trajetória média da população (fixa) e as trajetórias aleatórias genético-aditivas e de ambiente permanente foram modeladas por funções paramétricas, como a logarítmica, de Ali \& Schaeffer (1987) e a exponencial de Wilmink (1987). A função de Ali \& Schaeffer (AS) é composta de cinco parâmetros e descrita por:

$$
\mathrm{y}=\mathrm{a}+\mathrm{bV}+\mathrm{c} \mathrm{V}^{2}+\mathrm{dZ}+\mathrm{e} \mathrm{Z}^{2}
$$

em que $\mathrm{V}=(\mathrm{t} / 305)$ e $\mathrm{Z}=\ln (305 / \mathrm{t})$

em que $a$ = parâmetro relacionado ao pico de produção; $b$ e $c=$ parâmetros relacionados à fase de declínio da curva; e $d$ e $e=$ relacionados à fase de aumento da produção até o pico.

A função de Wilmink (WIL) é composta de três parâmetros e descrita pela fórmula:

$$
y=a+b t+c e^{(-0,05 t)}
$$

em que $a, b$ e $c=$ parâmetros da função associados, respectivamente, à produção inicial de leite, à taxa de declínio da produção de leite após o pico de lactação e à taxa de ascensão da produção de leite até atingir o pico de lactação. Para ambas as funções, $\mathrm{t}$ é o número de dias em lactação.

$\mathrm{Na}$ forma matricial, o modelo de regressão aleatória pode ser representado por:

$$
\mathrm{y}=\mathrm{Xb}+\mathrm{Za}+\mathrm{Wap}+\mathrm{e}
$$

em que: $\mathrm{y}=$ vetor das $\mathrm{N}$ observações, medidas em $\mathrm{N}_{\mathrm{d}}$ animais com registros; $b=$ vetor de efeitos fixos que inclui as soluções para GC e para as co-variáveis idade da vaca ao parto e dias em lactação; a, ap = vetores de soluções para os coeficientes de regressão aleatórios genético-aditivos e de ambiente permanente; e e $=$ vetor dos $\mathrm{N}$ diferentes resíduos; $\mathrm{X}, \mathrm{Z}, \mathrm{W}=$ matrizes de incidência para os efeitos fixos e os aleatórios genético direto e de ambiente permanente, respectivamente.

As pressuposições em relação aos componentes do modelo são:

$$
\mathrm{E}\left[\begin{array}{c}
\mathrm{y} \\
\mathrm{a} \\
\mathrm{ap} \\
\mathrm{e}
\end{array}\right]=\left[\begin{array}{c}
\mathrm{Xb} \\
0 \\
0 \\
0
\end{array}\right] ; \mathrm{e} \begin{aligned}
& \left.\mathrm{V}(\mathrm{a})=\mathrm{K}_{\mathrm{A}} \otimes \mathrm{Ap}\right)=\mathrm{K}_{\mathrm{AP}} \otimes \mathrm{I}_{\mathrm{Nd}} \\
& \mathrm{V}(\mathrm{e})=\mathrm{R}
\end{aligned}
$$

em que: $\mathrm{K}_{\mathrm{A}}$ e $\mathrm{K}_{\mathrm{AP}}=$ matrizes de co-variâncias entre os coeficientes de regressão aleatórios genético-aditivos e de ambiente permanente, respectivamente; $\mathrm{A}=$ matriz de parentesco entre os indivíduos; $\mathrm{I}_{\mathrm{Nd}}=$ matriz identidade; $\mathrm{R}=$ matriz contendo as variâncias residuais.

Os componentes de variância foram estimados pelo método da Máxima Verossimilhança Restrita (REML), usando a opção DXMRR do pacote estatístico DFREML (Meyer, 1998). A comparação entre os modelos de regressão aleatória foi feita usando os Critérios de Informação de Akaike (AIC) e Bayesiano de Schwarz (BIC), como relatado por Wolfinger (1993).

Os resultados obtidos pelos modelos de regressão aleatória foram comparados aos resultados de análises realizadas por modelos padrão, de dimensão finita (TDMO). No mesmo conjunto de dados, foram realizadas análises bicaracterísticas entre as PLDC com a produção de leite acumulada até os 305 dias (P305). Para as PLDC, utilizou-se um modelo animal que incluiu os efeitos fixos de grupo de contemporâneos (rebanho, ano e mês do controle), classe de idade da vaca ao parto, co-variável dias em lactação 
(regressão linear) e efeito aleatório genético aditivo. Para a P305, foram incluídos no modelo o mesmo efeito aleatório e os efeitos fixos de grupo de contemporâneos, definido por rebanho, ano e estação do parto, e a idade da vaca ao parto como co-variável, efeitos linear e quadrático.

Depois de escolhido o melhor modelo de regressão aleatória, foram preditos os valores genéticos para os coeficientes de regressão aleatória para cada animal, usados para predizer valores genéticos para a produção de leite em cada dia da lactação $\left(V_{i j}\right)$. Considerando a função AS, os valores genéticos foram calculados da seguinte forma:

$\mathrm{VG}_{\mathrm{ij}}=\mathrm{VG}_{0(\mathrm{j})}+\mathrm{VG}_{1(\mathrm{j})} \cdot \mathrm{t} / 305+\mathrm{VG}_{2(\mathrm{j})} \cdot(\mathrm{t} / 305)^{2}+$ $V_{3(j)} \cdot \ln (305 / t)+V_{4(j)} \cdot \ln (305 / t)^{2}$, em que: $V_{0} a_{0} V_{4}$ são os valores genéticos para os coeficientes de regressão aleatórios para cada animal. Após o cálculo dos valores genéticos diários, foram calculados os valores genéticos para períodos específicos da lactação: MRA305 é a somatória dos valores genéticos por todo o período da lactação; MRA60-305 é a média dos valores genéticos a partir dos 60 dias até o final da lactação; MRA100 é a média dos valores genéticos nos primeiros 100 dias de lactação; MRA200 é a média dos valores genéticos no período de 100 a 200 dias de lactação; MRA300 é a média dos valores genéticos no período de 200 a 300 dias de lactação; MRA60 é a média dos valores genéticos nos primeiros 60 dias de lactação, correspondentes ao período do pico da lactação.

Os critérios mencionados anteriormente, principalmente as médias de valores genéticos em períodos após o pico da lactação, poderiam ser usados como critérios de seleção para a persistência da lactação, como MRA200, MRA300 e MRA60-305.

Os valores genéticos preditos para os critérios propostos para o MRA foram comparados aos valores genéticos preditos pelo modelo tradicionalmente usado para avaliar a produção de leite (PTA305). Assim, foram estimadas as correlações de posto entre os valores genéticos para todos os animais e para touros com filhas.

\section{Resultados e Discussão}

A produção de leite média no dia do controle foi de $8,57 \mathrm{~kg}$, com desvio-padrão de $3,11 \mathrm{~kg}$ e coeficiente de variação de $36,28 \%$. Houve aumento da produção (Tabela 1) nos primeiros dois meses até chegar ao pico, que ocorreu próximo aos 60 dias $(10,32 \mathrm{~kg})$. A partir daí, a produção decresceu gradualmente até o final da lactação $(6,42 \mathrm{~kg})$.

Para os modelos de regressão aleatória, os valores de Log L melhoraram à medida que o número de parâmetros dos modelos aumentou (Tabela 2). De acordo com os resultados dos testes para comparação dos modelos, AIC e BIC, os modelos contendo homogeneidade de variâncias residuais promoveram os piores ajustes, independentemente da função empregada para modelar as trajetórias genéticas e de ambiente permanente. É necessário, portanto, considerar uma estrutura de variâncias heterogêneas para o resíduo, uma vez que as variâncias mostraram comportamento diferenciado no decorrer da lactação. Do mesmo modo, Cobuci et al. (2007), utilizando registros de lactações de vacas da raça Holandesa, empregaram dois modelos de regressão aleatória, considerando homogeneidade e heterogeneidade de variâncias, com dez classes distintas. Esses autores concluíram que o MRA contendo homogeneidade proporcionou partição incorreta dos efeitos genéticos e ambientais.

Para ambas as funções paramétricas empregadas neste estudo, os valores de AIC indicaram que a melhor estrutura de variâncias residuais foi aqueal contendo seis variâncias heterogêneas, enquanto o BIC indicou modelos contendo quatro variâncias. Como o teste BIC tende a penalizar modelos mais parametrizados, sendo mais rigoroso que o AIC, a estrutura de variâncias residuais contendo quatro classes heterogêneas seria suficiente para explicar a variação desse efeito no decorrer da lactação. Pelo Log L, quando o número de classes de variâncias residuais aumentou, mesmo havendo aumento no máximo da função, esse aumento foi pouco significativo.

Tabela 1 - Produção de leite de acordo com o mês do controle e o dia em lactação

\begin{tabular}{|c|c|c|c|c|c|}
\hline Mês & Dias em lactação & $\mathrm{N}^{\circ}$ de observações & Média (kg) & DP & $\mathrm{CV}(\%)$ \\
\hline 1 & $5-30$ & 1285 & 9,80 & 3,20 & 35,22 \\
\hline 2 & $31-60$ & 1700 & 10,32 & 3,63 & 31,00 \\
\hline 3 & $61-90$ & 1652 & 9,77 & 3,49 & 35,77 \\
\hline 4 & $91-120$ & 1645 & 9,55 & 3,59 & 37,68 \\
\hline 5 & $121-150$ & 1480 & 9,23 & 3,46 & 37,53 \\
\hline 6 & $151-180$ & 1436 & 8,49 & 3,15 & 37,09 \\
\hline 7 & $181-210$ & 1396 & 7,92 & 2,89 & 36,47 \\
\hline 8 & $211-240$ & 1295 & 7,43 & 2,71 & 36,59 \\
\hline 9 & $241-270$ & 1200 & 6,83 & 2,55 & 37,46 \\
\hline 10 & $270-305$ & 946 & 6,42 & 2,43 & 37,99 \\
\hline
\end{tabular}


Das duas funções de regressão paramétricas usadas neste estudo (Tabela 2), de acordo com os valores do Log Le pelos testes AIC e BIC, a AS foi superior à de WIL, o que pode ser explicado pelo maior número de parâmetros desta função em comparação à de WIL. Assim, de acordo com os critérios avaliados, o melhor modelo para explicar as trajetórias genética e de ambiente permanente, seria o AS, associado a uma estrutura de variâncias residuais, com quatro classes heterogêneas.

Para a função de WIL, tanto para o efeito genético aditivo quanto de ambiente permanente, as maiores variâncias estiveram associadas aos parâmetros b e c; para ambiente permanente, os valores foram sempre maiores. As correlações entre estes dois coeficientes de regressão aleatória foram maiores que as demais, de $-0,93$ para o efeito aditivo e de -0,95, para ambiente permanente. Esses valores indicam relação forte entre a taxa de declínio (b) e a de ascensão da produção de leite até atingir o pico de lactação (c). As correlações da função AS para o efeito genético aditivo foram maiores entre os parâmetros a e b $(-0,93)$; a e d $(-0,93)$; d com e $(-0,89)$.

As variâncias fenotípicas, genéticas e ambientais para as PLDC, estimadas para os MRA empregando as funções paramétricas WIL e AS para modelar as trajetórias aleatórias, descrevem a forma da curva de lactação (Figura 1), com os valores atingindo um máximo entre o segundo e o terceiro mês de lactação (no caso da função AS) e decrescendo até o final do período. No caso da função WIL, o pico das variâncias foi atingido para produção já no primeiro controle, tendendo a superestimar as variâncias nesse período. Os componentes de variância estimados pelo modelo de dimensão finita (TDMO) também foram apresentados para comparação. Para ambas as funções paramétricas empregadas neste estudo, as variâncias mostraram a forma da curva de lactação, com os valores atingindo um máximo próximo entre o segundo e o terceiro mês (no caso da função AS), decrescendo até o final da lactação. No caso da função WIL, o pico das variâncias foi atingido já no primeiro controle, tendendo a superestimar as variâncias nesse período.

As variâncias fenotípicas para a função AS foram muito próximas às estimadas nas análises bicaracterísticas (TDMO) e apresentaram o mesmo comportamento no decorrer da lactação. Empregando a função de WIL, as variâncias fenotípicas foram superestimadas no início da lactação.

Os modelos de regressão aleatória tenderam a estimar os valores para as variâncias genéticas maiores que o TDMO (Figura 1). Para a função AS, a trajetória mostrou aumento nas variâncias genéticas para a produção de leite até no terceiro controle e depois declinou até o final da lactação. As variâncias para a função de Wilmink apresentaram a mesma tendência das variâncias fenotípicas e foram maiores que as estimadas pelo TDMO na maior parte da lactação, com exceção do sexto e décimo controles.

As variâncias ambientais (Figura 1) são representadas pela somatória das variâncias de ambiente permanente e temporário para o MRA. Os modelos de regressão aleatória tiveram variâncias ambientais menores que as estimadas pelo TDMO do terceiro ao quinto controle. Ambas as funções paramétricas (AS e WIL) apresentaram tendência muito parecida para as variâncias no decorrer da lactação, exceto para a produção do primeiro controle.

As estimativas de herdabilidade para as PLDC estimadas pelas análises bicaracterísticas utilizando-se modelos de dimensão finita (TDMO) variaram de 0,14 a 0,34 e oscilaram por todo o período da lactação (Figura 2). As maiores estimativas foram obtidas para as produções do primeiro e do sexto controles. Para a produção acumulada até os 305

Tabela 2 - Número de variâncias residuais (e), número de parâmetros (p), função de verossimilhança (2log L) e critérios de informação de Akaike (AIC) e Bayesiano de Schwarz (BIC) para os modelos de regressão aleatória obtidos utilizando-se as funções paramétricas de Wilmink e de Ali e Schaeffer para as trajetórias genéticas e de ambiente permanente

\begin{tabular}{lcccc}
\hline Modelo & $\mathrm{e}$ & $\mathrm{p}$ & Alog $\mathrm{AIC}$ & \\
\hline & & & WIL & \\
1 & 1 & 13 & -14.496 & 29.019 \\
2 & 4 & 16 & -14.404 & 28.841 \\
3 & 6 & 18 & -14.398 & 28.832 \\
4 & 10 & 22 & -14.396 & 28.836 \\
\hline & & & AS & 28.968 \\
5 & 1 & 31 & -14.249 & 28.561 \\
6 & 4 & 34 & -14.225 & 28.518 \\
7 & 6 & 36 & -14.221 & 28.795 \\
8 & 10 & 40 & -14.220 & 28.514 \\
\hline
\end{tabular}

*Valores em negrito indicam o melhor modelo com base em AIC e BIC. 
Tabela 3 - Estimativas de variâncias (diagonal), co-variâncias (abaixo da diagonal) e correlações (acima da diagonal) para os coeficientes de regressão aleatória empregando as funções paramétricas de Wilmink e Ali e Schaeffer para os efeitos genético aditivo e de ambiente permanente, respectivamente, e uma estrutura de variâncias residuais contendo quatro classes

\begin{tabular}{|c|c|c|c|c|}
\hline $\mathrm{a}$ & $\mathrm{b}$ & $\mathrm{c}$ & d & $\mathrm{e}$ \\
\hline \multicolumn{5}{|c|}{ Wilmink } \\
\hline \multicolumn{5}{|c|}{ Efeito genético aditivo } \\
\hline 2,89 & $-0,42$ & 0,12 & & \\
\hline$-3,11$ & 18,70 & $-0,93$ & & \\
\hline 0,76 & $-15,22$ & 14,38 & & \\
\hline \multicolumn{5}{|c|}{ Efeito de ambiente permanente } \\
\hline 5,30 & $-0,58$ & 0,40 & & \\
\hline$-9,84$ & 53,45 & $-0,95$ & & \\
\hline 5,58 & $-42,25$ & 37,16 & & \\
\hline
\end{tabular}

Efeito genético aditivo

$\begin{array}{rrrrr}49,03 & -0,93 & 0,37 & -0,93 & 0,70 \\ -57,37 & 76,95 & -0,66 & 0,78 & -0,45 \\ 9,06 & -20,18 & 12,00 & -0,06 & -0,36 \\ -23,37 & 24,47 & -0,70 & 12,91 & -0,89 \\ 2,91 & -2,35 & -0,75 & -1,90 & 0,35\end{array}$

Efeito de ambiente permanente

\begin{tabular}{rrrrr}
20,64 & $-0,37$ & $-0,15$ & $-0,45$ & 0,12 \\
$-15,98$ & 91,76 & $-0,85$ & $-0,58$ & 0,78 \\
$-6,30$ & $-72,84$ & 80,66 & 0,92 & $-0,95$ \\
$-8,72$ & $-23,91$ & 35,41 & 18,36 & $-0,93$ \\
0,82 & 11,01 & $-12,56$ & $-5,83$ & 2,16 \\
\hline
\end{tabular}

dias (P305), a herdabilidade média de todas as análises bicaracterísticas entre as produções no dia do controle e a P305 foi de 0,27.

As estimativas de herdabilidade para ambos os modelos de regressão aleatória descreveram o mesmo comportamento durante a maior parte da lactação, com exceção do primeiro e último controles, cujas herdabilidades foram superiores quando a função AS foi empregada. As maiores estimativas ocorreram para as produções da primeira metade da lactação. Com a função AS, as estimativas de herdabilidade variaram de 0,33 (primeiro mês) e decresceram com o avanço da lactação até o valor de 0,21 (nono mês). No meio da lactação, esses valores foram próximos a 0,30 . Os valores estimados pela função de Wilmink variaram de 0,30 (segundo mês) a 0,17 (décimo mês). Nos pontos extremos da lactação, a função AS apresentou estimativas próximas às obtidas pelo TDMO. Costa et al. (2002), trabalhando com dados da mesma raça, encontraram estimativas de herdabilidade mais altas, variando de 0,71 (início) a 0,27 (final da lactação). Araújo (2003), empregando a função de WIL para modelar
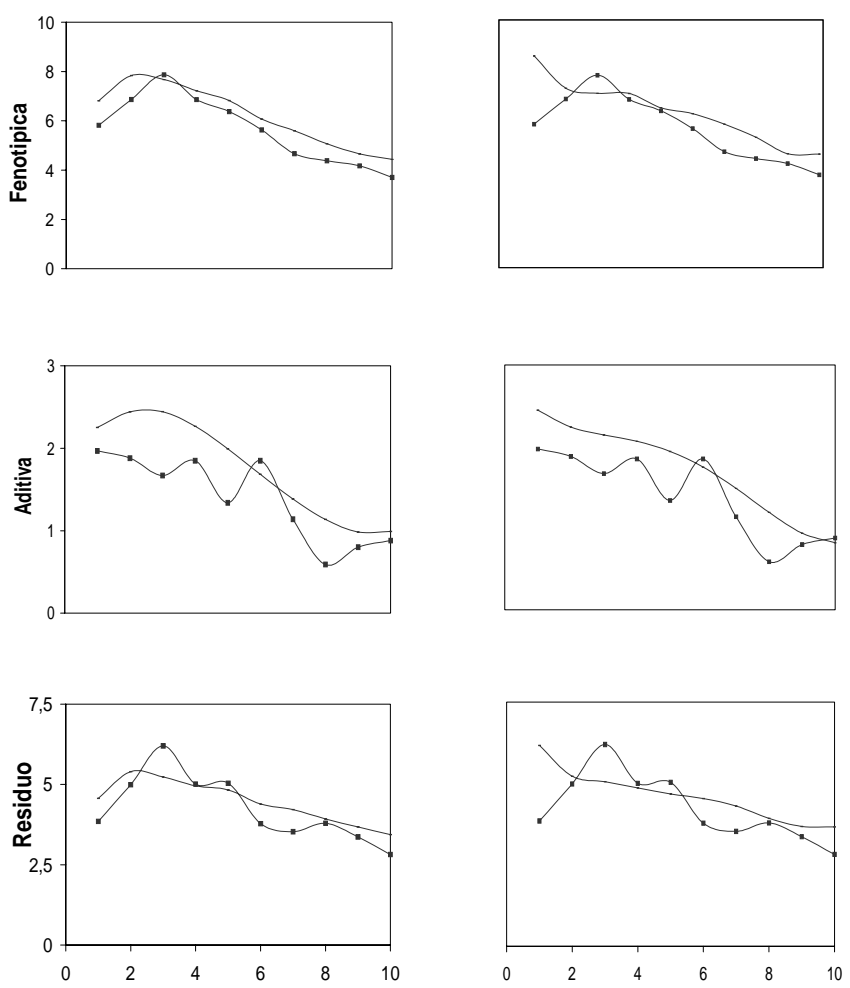

as trajetórias aleatórias na raça Holandesa, encontrou estimativas que variaram de 0,15 a 0,31 , maiores no meio e no final da lactação.

As superfícies representando as correlações genéticas, fenotípicas e de ambiente permanente de animal (Figura 3) indicaram a mesma tendência das correlações estimadas para as duas funções paramétricas empregadas para modelar as produções de leite no dia do controle. Independentemente da função utilizada, as correlações genéticas entre as produções de leite foram menores conforme aumentou o intervalo dos controles, apresentando valores próximos à unidade nos períodos adjacentes. Estes resultados concordam com os relatados por Rekaya et al. (1999) e Olori et al. (1999), para a raça Holandesa, por Kettunen et al. (2000) para a raça Ayrshire e, por Costa et al. (2002), para a raça Gir.

Todas as estimativas para as correlações genéticas foram positivas e superiores a 0,42 , indicando que a seleção para o aumento da produção de leite em determinado ponto da lactação terá reflexo positivo sobre todos os outros pontos da lactação. Os resultados deste estudo diferem 

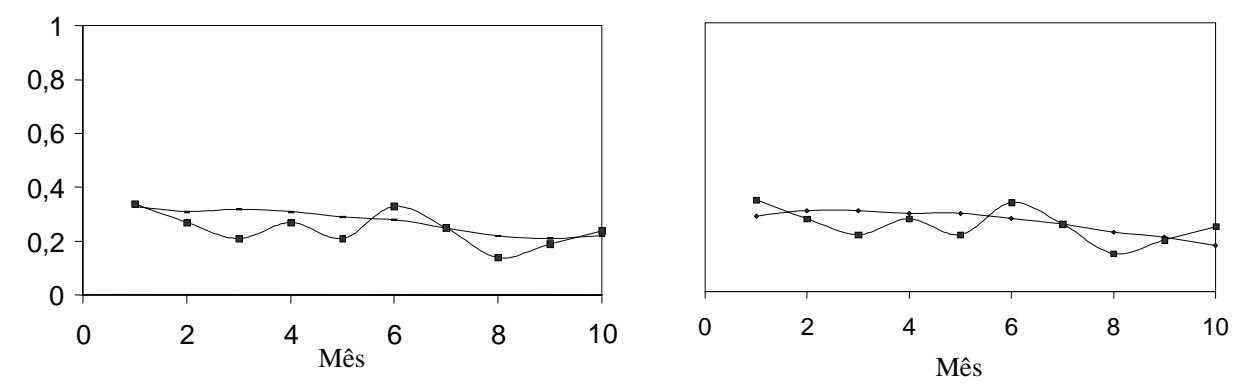

Figura 2 - Estimativas de herdabilidade para as produções de leite mensais, de acordo com o modelo de dimensão finita (- $\mathbf{\square}$-) e com os modelos de regressão aleatória (-), obtidas aplicando-se as funções paramétricas de Ali e Schaeffer (esquerda) e de Wilmink (direita) para ajustar as trajetórias aleatórias.
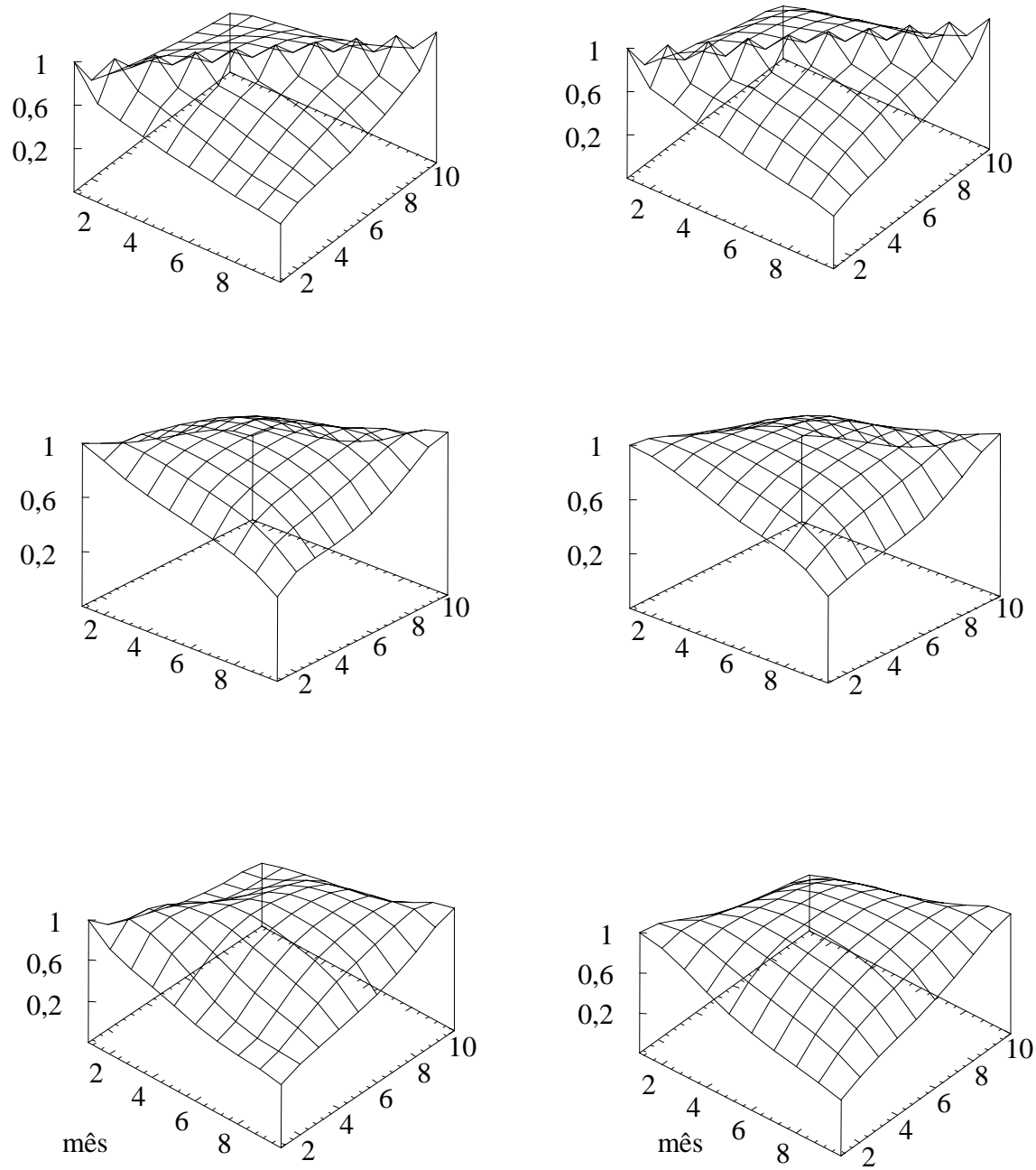

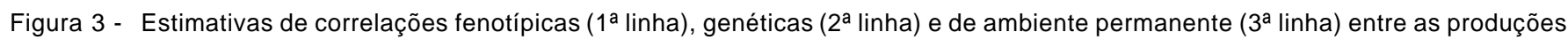
mensais, de acordo com as funções paramétricas de Ali e Schaeffer (à esquerda) e Wilmink (à direita), para modelar as trajetórias aleatórias. 
Tabela 4 - Valores genéticos preditos para as produções de leite em diferentes períodos da lactação aplicando-se o MRA e o modelo padrão (PTA305) para todos os touros

\begin{tabular}{|c|c|c|c|c|c|}
\hline Variável & № de touros & Média $(\mathrm{kg})$ & Desvio-padrão (kg) & Mínimo & Máximo \\
\hline MRA305 & 871 & 37,49 & 208,14 & $-452,88$ & 1217,00 \\
\hline MRA60 & 871 & 0,10 & 0,81 & $-2,82$ & 4,08 \\
\hline MRA 100 & 871 & 0,12 & 0,85 & $-2,54$ & 4,40 \\
\hline MRA 200 & 871 & 0,14 & 0,78 & $-1,76$ & 4,87 \\
\hline MRA 300 & 871 & 0,10 & 0,49 & $-1,36$ & 2,81 \\
\hline MRA60-305 & 871 & 31,40 & 165,01 & $-393,77$ & $1.004,00$ \\
\hline PTA305 & 871 & 25,74 & 174,83 & $-643,31$ & 908,04 \\
\hline
\end{tabular}

Tabela 5 - Valores genéticos preditos para as produções de leite em diferentes períodos da lactação aplicando-se o MRA e o modelo padrão (PTA305) para todos os animais

\begin{tabular}{lccrrr}
\hline Variável & № de animais & Média $(\mathrm{kg})$ & Desvio-padrão $(\mathrm{kg})$ & Mínimo & Máximo \\
\hline MRA305 & 5.711 & 66,04 & 241,91 & $-527,60$ & $-2,82$ \\
MRA60 & 5.711 & 0,22 & 0,90 & $-2,54$ & 4,39 \\
MRA100 & 5.711 & 0,25 & 0,96 & $-1,91$ & $-1,50$ \\
MRA200 & 5.711 & 0,25 & 0,92 & $-414,55$ \\
MRA300 & 5.711 & 0,14 & 0,58 & 3,51 \\
MRA60-305 & 5.711 & 52,73 & 193,70 & $-832,79$ & $1.150,00$ \\
PTA305 & 5.711 & 41,62 & 203,99 & 992,46 \\
\hline
\end{tabular}

dos encontrados por Costa et al. (2002), também com produção de leite no dia do controle da raça Gir no Brasil. Os autores obtiveram estimativas de correlações genéticas negativas entre os extremos da curva de lactação quando usaram a função paramétrica de Wilmink ou polinômios de Legendre para modelar as trajetórias aleatórias.

Brotherstone et al. (2000), utilizando as mesmas funções paramétricas usadas neste estudo, para gado Holandês, observaram estimativas negativas entre o primeiro controle e os controles a partir dos 42 dias de lactação e as atribuíram ao fato de que funções paramétricas não modelam a associação entre as produções no início e no fim da lactação. Cobuci (2005), por sua vez, não obteve estimativas de correlações genéticas negativas quando utilizou a função de Wilmink para os efeitos genético aditivo e de ambiente permanente. Contudo, quando o autor utilizou um modelo que considerava a variância de ambiente permanente constante, ocorreram estimativas negativas de correlações genéticas.

Os valores genéticos para os touros com filhas (Tabela 4) e para todos os animais (Tabela 5) foram preditos apenas para o MRA que empregou a função AS, uma vez que essa função apresentou os melhores ajustes, segundo os testes e as estimativas dos parâmetros foram mais próximas ao modelo padrão (TDMO). As médias dos valores genéticos preditos para MRA305 e PTA305 foram próximas, embora as médias para o MRA tenham sido pouco maiores. Entretanto, a variação dos valores genéticos para MRA305 foi menor que para PTA305, o que foi observado para todos os animais ou para os touros. A variação das médias dos valores genéticos para os touros foi muito grande, o que mostra a diversidade do potencial genético dos animais provenientes dos diferentes rebanhos.

As correlações entre MRA305 e todas as características que foram geradas pelo MRA foram próximas a 0,90 , tanto para touros como para todos os animais (Tabela 6). Estas correlações indicam que deve haver coincidência na classificação dos animais, usando-se como critério de seleção qualquer uma das medidas. Entretanto, as correlações de posto entre os valores genéticos para medidas do início da lactação (MRA60 e MRA100), com a medida que representa o final da lactação (MRA300), foram menores. Por exemplo, entre MRA100 com MRA300 a correlação foi de 0,67 para touros e de 0,77 para todos os animais. Entre MRA60, que representa a produção em volta do pico, com MRA300 foi de 0,59 (touros) e de 0,70 (todos os animais). A medida MRA300 expressa a média dos valores genéticos de um animal no período de 200 a 300 dias de lactação, o que, para a raça Gir, é um período em que muitos animais já tiveram suas lactações encerradas em razão da baixa produção, principalmente em se tratando de primeiras lactações. As correlações de MRA60 e MRA100 com MRA60-305 foram maiores que com MRA300 e próximas a 0,80. A característica MRA60-305 expressa a média dos valores genéticos para as produções de leite no período após os 60 dias de lactação e, como na sua composição entram os valores genéticos anteriores aos 200 dias, o efeito de baixos valores genéticos acabam diluídos no final da lactação (de 200 a 300 dias), contrário ao que ocorre com MRA300. 
Tabela 6 - Correlação de posto entre os critérios de seleção para touros com progênie (diagonal inferior) e para todos os animais (diagonal superior)

\begin{tabular}{lccccccc}
\hline Critério & MRA305 & MRA60 & MRA100 & MRA200 & MRA300 & MRA60-305 & PTA305 \\
\hline MRA305 & & 0,90 & 0,95 & 0,99 & 0,91 & 0,99 & 0,74 \\
MRA60 & 0,88 & & 0,99 & 0,87 & 0,70 & 0,85 & 0,91 \\
MRA100 & 0,93 & 0,90 & & 0,92 & 0,77 & 0,99 & 0,73 \\
MRA200 & 0,98 & 0,83 & 0,89 & & & 0,95 & 0,76 \\
MRA300 & 0,87 & 0,59 & 0,67 & 0,88 & 0,93 & 0,75 \\
MRA60-305 & 0,98 & 0,80 & 0,87 & 0,99 & 0,68 & 0,66 \\
PT A305 & 0,65 & 0,53 & 0,56 & 0,63 & & \\
\hline
\end{tabular}

As correlações de posto entre os valores genéticos, para as características geradas pelo MRA com a PTA305, foram sempre menores que 0,76 (para todos os animais) e menores que 0,68 (para os touros), sugerindo, portanto, que podem ocorrer mudanças consideráveis na classificação dos animais se estas características vierem a ser adotadas como critérios de seleção alternativos para a raça Gir. A adoção de MRA305 poderia auxiliar na seleção de animais com maiores persistências da lactação, uma vez que representa a somatória dos valores genéticos para as produções de leite em toda a lactação, que são, na verdade, os desvios da curva de lactação fixa. Assim, animais com curvas genéticas com maiores desvios em todo o período da lactação seriam os mais persistentes. Entretanto, esse critério não assegura que exista um equilíbrio destes valores genéticos nas diferentes fases e, características como MRA200, MRA300 e MRA60-305 poderiam auxiliar nesse sentido.

Os gráficos de dispersão dos valores genéticos preditos para os touros, comparando MRA305 e PTA305 com os demais critérios (Figura 4), enfatizam a relação já mostrada na Tabela 6. Menor dispersão nos valores genéticos ocorreu
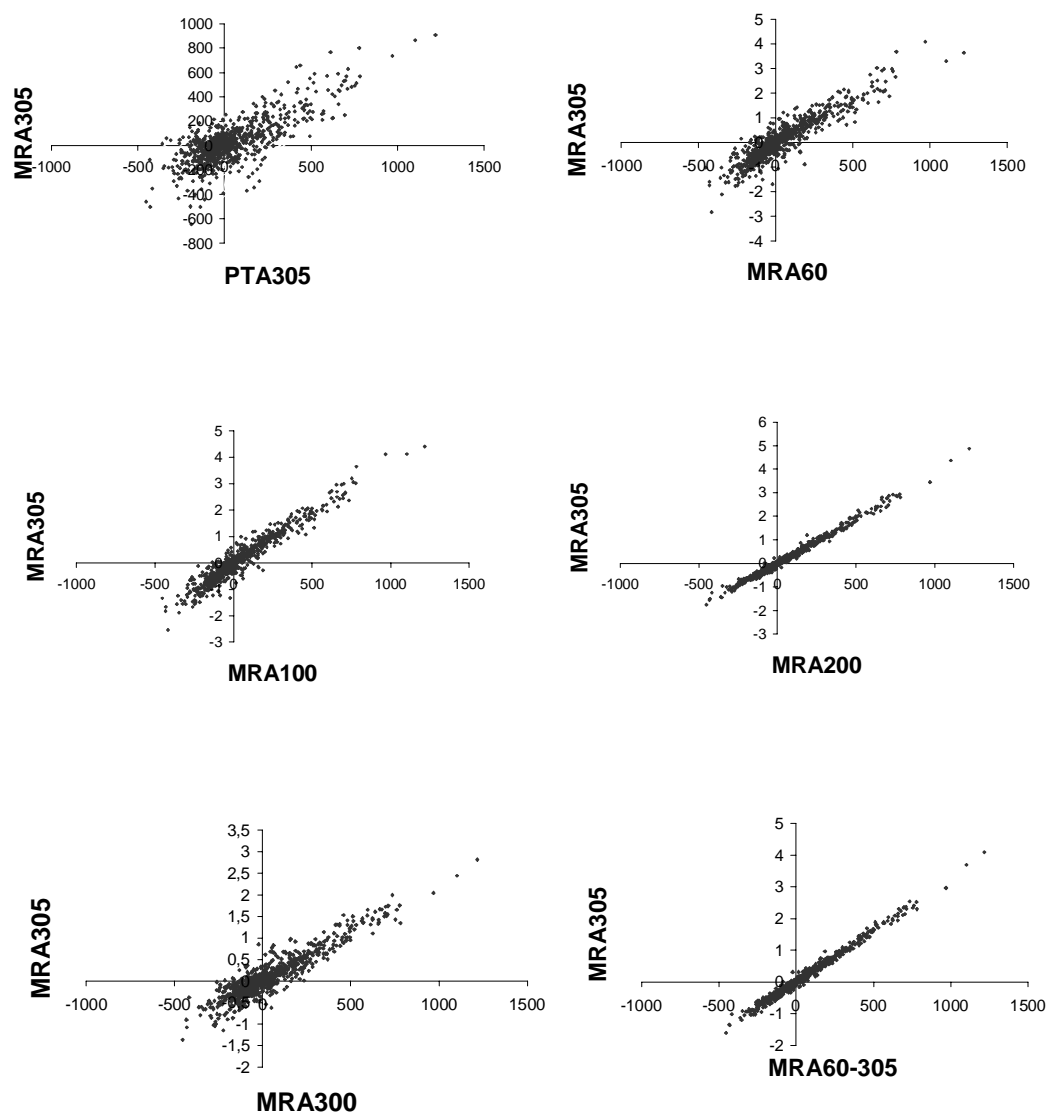

Figura 4 - Dispersão dos valores genéticos preditos para a produção acumulada até os 305 dias pelo método tradicional (PTA305) e por regressão aleatória (MRA305) e em diferentes períodos da lactação por meio de MRA. 
quando foram comparados MRA305 com MRA200 e com MRA60-305. Para as demais características, ocorreu dispersão desses valores, apesar de as tendências indicarem sempre relação linear e positiva. Quando os valores genéticos preditos pelo método tradicional (PTA305) foram comparados aos preditos pelo MRA (Figura 5), houve grande dispersão na distribuição dos valores genéticos, apesar de as tendências serem lineares e positivas.

Os estudos propondo novos critérios de seleção para produção de leite aplicando os modelos de regressão aleatória são ainda escassos na literatura. Pesquisadores de países como Canadá, Holanda e Finlândia propuseram algumas medidas de persistência. Essas medidas sempre foram expressas pela subtração entre as médias dos valores genéticos do final da lactação pela média dos valores genéticos no início da lactação. Kistemaker (2003) encontrou correlações muito baixas entre os valores genéticos preditos por estas medidas e para a PTA305, próximas a 0,10.

É importante verificar que, como os MRA proporcionam a predição de valores genéticos para todos os pontos da lactação e esses valores são expressos como desvios de uma média populacional, os animais com maiores valores genéticos acumulados na lactação seriam os mais desejáveis, assim como os animais com maiores valores genéticos médios em períodos específicos ou críticos da lactação, por exemplo, após os 200 dias da lactação.
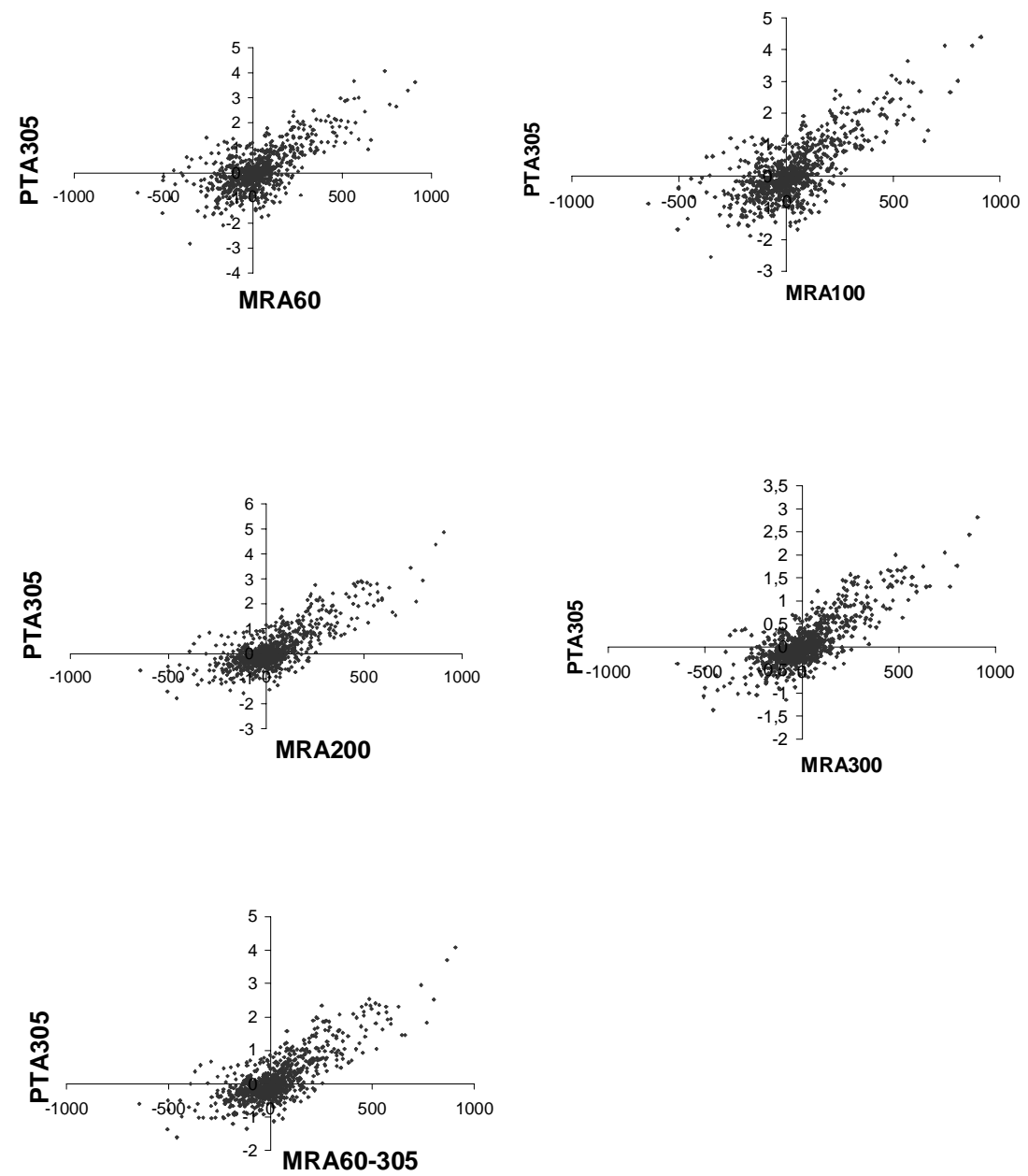

Figura 5 - Dispersão dos valores genéticos preditos para a produção acumulada até os 305 dias pelo método tradicional (PTA305) e para diferentes períodos da lactação por meio de MRA.

\section{Conclusões}

É necessário utilizar heterogeneidade de variâncias residuais para modelar a produção de leite usando os modelos de regressão aleatória neste estudo. A função de AS foi suficiente para descrever as trajetórias aleatórias genética aditiva e de ambiente permanente. Os resultados sugerem diferenças na classificação dos animais quando os 
critérios de seleção são baseados no valor predito para a produção acumulada até os 305 dias pelo método tradicional e as medidas geradas pelo modelo de regressão aleatória. A somatória dos valores genéticos preditos por todo o período da lactação usando modelos de regressão aleatória poderia ser usada como critério de seleção para identificação de animais superiores para a produção acumulada até os 305 dias e, também, para a persistência da lactação. As medidas baseadas na média dos valores genéticos no período de 200 a 300 dias (MRA300) e de 60 a 305 dias (MRA60-305) poderiam ser adotadas como critério de seleção, mas a MRA300 pode dar maior ênfase para a produção de leite no terço final da lactação e também para a persistência.

\section{Literatura Citada}

ALI, T.E.; SCHAEFFER, L.R. Accounting for covariances among test day milk yields in dairy cows. Journal of Animal Science, v.67, n.2, p.637-644, 1987.

ARAÚJO, C.V. Modelos de regressão aleatória para avaliação genética da produção de leite na raça Holandesa. Viçosa, MG: Universidade Federal de Viçosa, 2003. 85p. Tese (Doutorado em Zootecnia) - Universidade Federal de Viçosa, 2003.

BIGNARDI, A.B. Estimação de parâmetros genéticos para produção de leite de primeiras lactações de vacas da raça Holandesa usando modelos de dimensão infinita. Jaboticabal: Universidade Estadual Paulista, 2006. 75p. Dissertação (Mestrado em Genética e Melhoramento Animal) - Universidade Estadual Paulista, 2006.

BROTHERSTONE, S.; WHITE, I.M.S.; MEYER, K. Genetic modeling of daily yields using orthogonal polynomials and parametric curves. Journal of Animal Science, v.70, n.2, p.407-415, 2000.

COBUCI, J.A.; EUCLYDES, R.F.; COSTA, C.N. et al. Genetic evaluation for persistency of lactation using random regression model. Genetics and Molecular Biology, v.30, p.349-355, 2007.

COBUCI, J.A.; EUCLYDES, R.F.; LOPES, P.S. et al. Estimation of genetic parameters for test-day milk in Holstein cows using a random regression model. Genetics and Molecular Biology, v.28, n.1, p.75-83, 2005.

COSTA, C.N.; MELO, C.M.R.; MACHADO, C.H.C. et al. Avaliação de funções polinomiais para ajuste da produção de leite no dia do controle de primeiras lactações de vacas Gir com modelo de regressão aleatória. In: REUNIÃO ANUAL DA SOCIEDADE BRASILEIRA DE ZOOTECNIA, 39., 2002, Recife, Pernambuco. Anais... Recife: Sociedade Brasileira de Zootecnia, 2002. (CD-ROM).

COSTA, C.N.; MELO, C.M.; MACHADO, C.H. et. al. Parâmetros genéticos para a produção de leite de controles individuais de vacas da raça Gir estimados com modelos de repetibilidade e regressão aleatória. Revista Brasileira de Zootecnia, v.34, n.5, p. 1520-1531, 2005.

EL FARO, L.; ALBUQUERQUE, L.G. Estimação de parâmetros genéticos para produção de leite no dia do controle e produção acumulada até 305 dias, para as primeiras lactações de vacas da raça Caracu. Revista Brasileira de Zootecnia, v.32, n.2, p.284-264, 2003.
HENDERSON JR., C.R. Analysis of covariance in the mixed model: higher level, non homogeneous, and random regressions. Biometrics, v.38, n.2, p.623-640, 1982.

JAMROZIK, J.; SCHAEFFER, L.R. Estimates of genetic parameters for a test day model with random regressions for production of first lactation Holsteins. Journal of Dairy Science, v.80, n.4, p.762-770, 1997.

JAMROZIK, J.; KISTEMAKER, G.J.; DEKKERS, J.C.M. et al. Comparison of possible covariates for use in a random regression model for analyses of test day yields. Journal of Dairy Science, v.80, n.10, p.2550-2556, 1997.

KETTUNEN, A.; MÄNTYSAARI, E.; PÖSÖ J. Estimation of genetic parameters for daily milk yield of primiparous Ayrshire cows by random regression test-day models. Livestock Production Science, v.66, n.1, p.251-261, 2000.

KISTEMAKER, G.J. [2003]. Comparison of persistence definitions in random regression test day models. Disponível em: http://www-interbull.slu.se/framesida-home.htm, publications). Acesso em: 28/4/2006.

LUDWICK, T.M.; PETERSEN, W.E. A measure of persistency of lactation of dairy cattle. Journal of Dairy Science, v.26, n.3, p.439-445, 1943

MEYER, K. DXMRR - a program to estimate covariance functions for longitudinal data by REML. In: WORLD CONGRESS OF GENETICS APPLIED TO LIVESTOCK PRODUCTION, 6., 1998, Armidale. Proceedings... Armidale: 1996. (CD-ROM).

OLORI, V.E.; HILL, W.G.; MCGUIRK, B.J. et al. Estimating variance components for test day milk records by restricted maximum likelihood with a random regression animal model. Livestock Production Science, v.61, n.1, p.53-63, 1999.

REKAYA, R.; CARABANO, M.J.; TORO, M.A. Random regression in animal models for test-day production in dairy cattle. In: WORLD CONGRESS ON GENETICS APPLIED TO LIVESTOCK PRODUCTION, 5., 1994, Guelph. Proceedings... Guelph: 1994. v.18, p.443-446.

SCHAEFFER, L.R.; DEKKERS, J.C.M. Random regressions in animal models for test-day production in dary cattle. In: WORLD CONGRESS ON GENETICS APPLIED TO LIVESTOCK PRODUCTION, 5., 1994, Guelph. Proceedings... Guelph: 1994. v.17, p.443-446.

SCHAEFFER, L.R. Tópicos avançados em melhoramento animal: random regression models (notas do curso). Jaboticabal: FCAV, 1996. p.25-33.

SCHAEFFER L.R. Application of random regression models in animal breeding. Livestock Production Science, v.16, n.4, p.335-348, 2004

SÖLKNER, J.; FUCHS, W. A comparison of different measures of persistency with special respect to variation of test-day milk yields. Livestock Production Science, v.16, n.3, p.305-319, 1987.

SWALVE, H.H. Genetic relationship between dairy lactation persistency and yield. Journal Animal Breeding Genetics, v.112, n.4, p.303-311, 1995.

TEKERLI, M.; AKINCI, Z.; DOGAN, I. et al. Factors affecting the shape of lactation curves of Holstein cows from the Balikesir province of Tukey. Journal of Dairy Science, v.83, n.6, p.1381-1386, 2000.

WOLFINGER, R. Covariance structure selection in general mixed models. Communications in Statistics, v.22, n.4, p.10791106, 1993.

WILMINK, J.B.M. Efficiency of selection for different cumulative milk, fat and protein yields in first lactation. Livestock Production Science, v.17, n.2, p.211-224, 1987. 